\title{
UNA MIRADA CRÍTICA A LA TEORÍA DEL SIGNO
}

\section{A critical look at the theory of the sign}

\section{Bartolo García Molina}

Doctor en Filosofía del Lenguaje por la Universidad Complutense de Madrid. Fue profesor en la Universidad Autónoma de Santo Domingo (UASD), Santo Domingo, República Dominicana, ORCID: org/0000-0001-6931-4823

Correo-e: bartologarciam@hotmail.com

\section{Recibido: $26 / 4 / 2020 \bullet$ Aprobado: 5/5/2020}

Cómo citar: García Molina, B. (2020). Una mirada crítica a la teoría del signo. Ciencia y Sociedad, 45(2), 65-77. Doi: https://doi. org/10.22206/cys.2020.v45i2.pp65-77

\section{Resumen}

En este estudio se reivindica el carácter multiplánico del signo lingüístico, el papel del mismo en todo proceso cognitivo y el acierto de Saussure al enfatizar la esencia psíquica o mental del significante. Si bien erró al descartar el aspecto material del signo lingüístico, la originalidad y agudeza de su acierto lo hace merecedor de un puesto cimero en la historia de la teoría del signo. También se revisa las teorías del signo de Charles Sanders, Louis Hjemslev y Charles Morris para formular una propuesta que recoge los aportes de los cuatro teóricos precedentes y reivindicar el objeto o referente del signo de Saussure, el aspecto mental tanto del significante como del significado; de Hjemslev, la sustancia de los dos planos del signo; y de Morris, el sujeto de la semiosis, el intérprete. A partir de la lectura de los cuatro teóricos objeto de este estudio y de los que componen y de los que componen el marco teórico se expone que solo Saussure y Hjemslev le dan un tratamiento realmente multiplánico al signo lingüístico; y que la imagen acústica o logogén del significante, y la imagen mental o concepto del significado tienen repercusiones en la didáctica, en la epistemología, en la filosofía del lenguaje y en la logopedia.

Palabras clave: signo; lenguaje de signos; semiología; lingüística; cambio lingüístico.

\section{Abstract}

In this study, the multiplanic character of the linguistic sign, its role in all cognitive processes and Saussure's success in emphasizing the mental or mental essence of the signifier are claimed. Although he erred in ruling out the material aspect of the linguistic sign, the originality and sharpness of his success makes him deserving of a top position in the history of sign theory. The theories of the sign of Charles Sanders, Louis Hjemslev and Charles Morris are also reviewed to formulate a proposal that gathers the contributions of the four preceding theorists and to vindicate the object or referent of Saussure's sign, the mental aspect of both the signifier and the signified; of Hjemslev, the substance of the two planes of the sign; and Morris, the subject of semiosis, the interpreter. From the reading of the four theorists object of this study and of those who compose and of those who compose the theoretical framework, it is exposed that only Saussure and Hjemslev give a truly multiplanic treatment to the linguistic sign; and that the acoustic or logogenic image of the signifier, and the mental image or concept of the meaning have repercussions in didactics, in epistemology, in the philosophy of language and in speech therapy.

Keywords: Sign; sign language; semiology; linguistics; linguistic change 
El concepto de signo puede revelarse tan fundamental para las ciencias del hombre como lo ha sido el de átomo, para las ciencias físicas; o el de célula, para las ciencias biológicas.

Charles Morris

\section{Introducción}

Desde Saussure se ha repetido que el signo lingüístico tiene dos planos o caras: significante y significado, aunque no siempre se ha explicado bien esos planos o caras del signo. Hay autores, incluso contemporáneos, que reducen el signo al significante. También hay autores como Louis Hjemslev, que sostienen el carácter dos veces biplánico del signo. A estos, se les suma las interpretaciones que se les da a esas teorías. Por ejemplo, para muchos, la teoría de Charles Sander Peirce es triádica; y para otros, es una mónada. Ante tanta vacilación, me planteé la siguiente pregunta: ¿cuántos planos tiene el signo lingüístico?

En este ensayo, defiendo la idea de que los dos planos del signo lingüístico propuestos por Saussure (significante y significado) se subdividen en dos subplanos, con lo cual me aproximo a la teoría de Hjemslev, pero también establezco las demarcaciones correspondientes con la misma. Trato de demostrar que el significante está compuesto por un aspecto material o tangible y otro aspecto inmaterial y abstracto (mental o virtual). Mi propuesta parte de las teorías del signo tanto de Saussure como de Hjemslev, aunque reviso las teorías de Charles Morris y Charles Sander Peirce. En todos los casos, establezco los puntos de coincidencia y diferencias.

Mi investigación tuvo como objetivo establecer cuántos planos tiene el signo lingüístico y separar los mitos de la realidad en torno a la teoría del signo, pues todavía hoy en las universidades, en los libros de texto, inclusive, en los libros especializados de lingüística y de semiótica se difunden apreciaciones inexactas de la teoría del signo en general, y muy particularmente de la teoría del signo propuesta por Saussure.
Mi estudio está enfocado desde la teoría del conocimiento, la lingüística cognitiva y la semiótica cuyo proceso de investigación consistió en revisar las cuatro propuestas fundamentales en torno al signo lingüístico. En orden cronológico: la de Charles Sander Peirce (1884) la de Ferdinand de Saussure (1906), la de Louis Hjemslev (1933) (1938) y la de Charles Morris. Luego de ponderar los aportes de cada uno, formulo mi propuesta. En ese mismo orden, presento los resultados de mis indagatorias y reflexiones.

\section{Una mirada a la teoría del signo de Peirce}

Peirce no formuló una teoría del signo lingüístico, sino todos los signos. Para él, un signo es:

... algo que para alguien representa o se refiere a algo respecto a un aspecto o carácter. Se dirige a alguien, esto es, crea en la mente de esa persona un signo equivalente, o, tal vez, un signo más desarrollado. El signo desarrollado es lo que yo llamo el representante del primer signo. El signo viene en lugar de algo, su objeto. Viene en lugar de ese objeto, no en todos sus aspectos, sino, solo con referencia a una especie de idea, que a veces he llamado el fundamento del representamen (Peirce, 1953, p. 228)

\section{Representamenn Objeto}

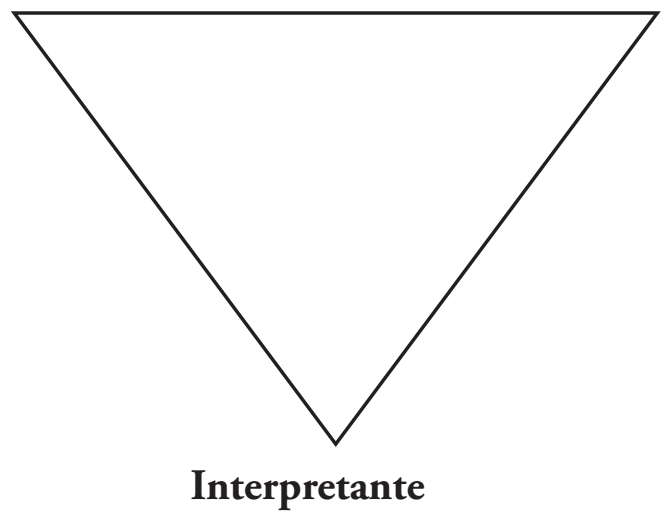

Figura 1. El esquema de la teoría del signo de Peirce (1973) 
El representamen es algo que está para alguien en lugar de algo, bajo un aspecto o capacidad. En la extensísima obra de Peirce, el representamen se presenta como el signo mismo. Con respecto a la teoría del signo de Saussure, el representamen se aproxima al componente material del significante (no incluye la imagen acústica, que es para Saussure el verdadero significante).

El representante es la imagen mental o concepto que media entre el representamen y el objeto. Son representaciones mentales que se forman los hablantes de un referente determinado. Es obvia la semejanza entre representamen y la imagen mental propuesta por Saussure.

El objeto es definido por Peirce como "....aquello a lo que el representamen se dirige" También Peirce afirma que el objeto: "Es aquello acerca de lo cual el signo (representamen) presupone un conocimiento para que sea posible proveer alguna información adicional sobre el mismo". (Peirce, 1973. p. 23)

La teoría del signo de Peirce se inserta en su epistemología de la primeridad, la segundidad y terceridad.

Un signo, o representamen, es un primero que está en relación genuina, triádica con un segundo, llamado objeto, de tal modo que es capaz que un tercero, llamado su representante asuma la misma relación triádica con su objeto que tiene el signo con el mismo objeto. (1973, p. 22).

Peirce presenta la primeridad como el modo de significación de lo que es tal como es, sin referencia a otra cosa. Para él, la primeridad es libertad, espontaneidad, originalidad, la posibilidad de que acontezca algo nuevo. La segundidad la concibe como el modo de significación de lo que es tal como es, con respecto a algo más, pero sin referencia a un tercer elemento. En cambio, la terceridad es “... el modo de significación de lo que es tal como es, a medida que trae un segundo y un tercer elemento (por ejemplo, un representamen y un objeto semiótico) y lo pone en correlación con el primero..." (1953, p. 328). La terceridad abarca la mediación, la síntesis de las categorías primeridad y segundidad lo que revela su importancia tanto en el proceso de percepción como de semiosis. "Por lo tanto, la terceridad marca el desarrollo vital de los signos. Es un proceso creador por medio del cual el caos se hace orden, y la confusión se hace claridad" (1953, p. 298). Saussure también le asigna un papel determinante a los signos y a la lengua en la organización de las percepciones y en la formación del pensamiento.

Psicológicamente, hecha abstracción de su expresión por medio de palabras, nuestro pensamiento no es más que una masa amorfa e indistinta. Filósofos y lingüistas han estado siempre de acuerdo en reconocer que, sin la ayuda de los signos, seríamos incapaces de distinguir dos ideas de manera clara y constante (...). Nada es distinto antes de la aparición de la lengua (Saussure, 1980, p. 140).

Se podría decir que el signo para Peirce es una mónada que entra en relación triádica con la base empírica del conocimiento (el objeto o realidad) y con la representación de la base empírica del conocimiento (imagen mental).

\section{Una mirada a la teoría del signo de Saussure}

Lo lógico sería esperar que Ferdinand de Saussure conectara su teoría del signo con la de su predecesor, Charles Sanders Peirce, ya que, para la década de 1890, cuando el primero comenzó a formular su teoría, ya el segundo lo había hecho. Sin embargo, no fue así a pesar de las coincidencias. No por falta de honestidad intelectual de Saussure, sino porque no la conocía. Baso mi afirmación en los siguientes datos: 
- Peirce publicó solo dos libros en tiempo de Saussure: Photometric Risearches (1878) y Studies of logic (1883); y varios papers de filosofía, lógica, filosofía de la ciencia, astronomía, topografía, física, matemática, religión, etc.

- Todavía en la última mitad del siglo XIX, no era común traducir artículos.

- La producción de Peirce era profusa, difusa y confusa.

- En la de Saussure, a Peirce se le conocía más como matemático, geodesta, químico, físico, filosófico, lógico.

- No hay evidencia de que Peirce y Saussure hayan tenido contacto físico o epistolar. El primero viajó varias veces a Europa, pero en ninguno de sus viajes participó en evento alguno de lingüística o de semiótica. El segundo, no visitó Estados Unidos.

- En Peirce, la teoría del signo forma parte de sus concepciones lógicas racionalistas; y su propuesta semiótica era más una teoría de conocimiento que de los sistemas de comunicación.

Por todo lo anterior, es justo atribuirle a Saussure una teoría diádica novedosa del signo, el cual definió como:

...la combinación del concepto y de una imagen acústica: pero en el uso corriente, este término designa generalmente la imagen acústica sola, por ejemplo: /árbol/ [árbol]. Se olvidan de que si llamamos signo a árbol no es más que gracias a que conlleva el concepto "árbol", de tal manera que la idea de la parte sensorial implica la del conjunto. (Saussure, 1980, p. 89).
La visión diádica del signo, Saussure la ilustra así:

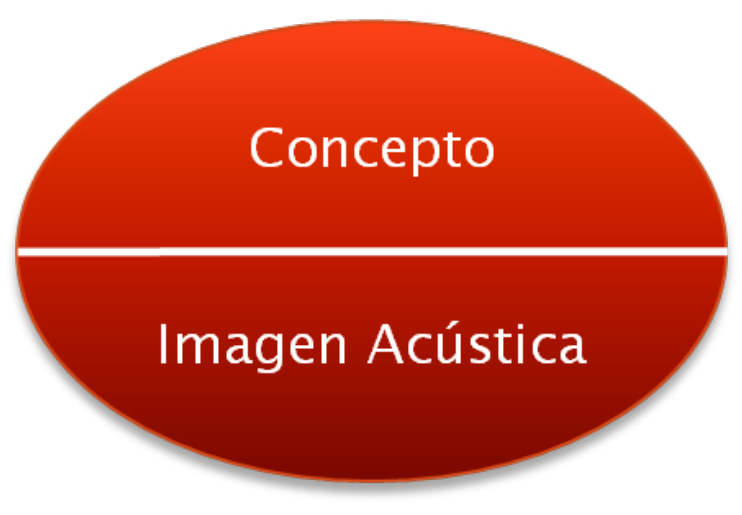

Figura 2. Concepción biplánica de Saussure del signo Fuente: Saussure, 1980, p. 89.

Saussure era consciente de que ya otros habían formulado una teoría del signo lingüístico. Pero observó que la concepción predominante en su tiempo reducía el signo al objeto material del significante o aspecto sensorial. "Se ha utilizado el término símbolo para designar el signo lingüístico, o, más exactamente, lo que nosotros llamamos el significante". (1980, p. 91). Saussure luego aclara que el significante no es el sonido, sino una especie de representación del aspecto sensorial o material, con lo que se va el extremo opuesto al de la tradición, pues niega la materialidad del significante.

Lo que el signo lingüístico une no es una cosa $y$ un nombre, sino un concepto y una imagen acústica. La imagen acústica no es el sonido material, cosa puramente física, sino su huella psíquica, la representación que de él nos deja el testimonio de nuestros sentidos (Sausssure, 1980, p. 91).

Saussure despoja de materialidad el significante, con lo que lo reduce a la representación acústica 
que cada hablante tiene de las palabras; en otros términos, para Saussure el significante es la conciencia fonológica de los hablantes de una lengua determinada.

Al desprender el significante de su base objetiva o material, y reducirlo a la conciencia fonológica, Saussure lo ubicó en la esfera de lo mental, o más específicamente de las representaciones acústicas. Con esto, en realidad, su afirmación de que se había utilizado el término símbolo para designar el signo lingüístico, o, más precisamente, lo que él llamaba significante, se hacía inexacta, pues las teorías vigentes del signo o de los símbolos no se referían a las imágenes acústicas, sino a la base material de las mismas, o sea, en caso del signo lingüístico, a los sonidos, los cuales son materiales o tangibles. A pesar de eso, se puede considerar un aporte extraordinario a la teoría del signo el haber observado e insistido que el significante también tiene un aspecto mental o abstracto, como lo postula hoy la fonología al encargarse de estudiar los sonidos ideales, que son las representaciones de los sonidos específicos que producen los hablantes de cualquier lengua natural.

A pesar de la insistencia de Saussure en el carácter psíquico del significante, hoy todavía predomina la visión materialista del significante. Bertil Malmberg es de los pocos seguidores de Saussure que comprendió y defendió el carácter psíquico o mental del significante. Malmberg (1974, p. 27) afirma que "El significante no es la misma onda sonora - hecho físico - sino la idea de la secuencia de sonidos, una imagen o una representación de aquel hecho".

En cuanto al significado, no cabe duda de su carácter abstracto o mental, pues Saussure lo explicó como si fuera equivalente a un concepto, o abstracción cognitiva (representante, para Peirce), que lógicamente tendría que hacerse a partir del referente, realidad u objeto peirciano. Claro, Saussure en su afán de despojar la lengua de materialidad, obvió que el significado existe en virtud de su relación con la realidad objetiva. "La lengua es una forma y no una sustancia” (1980, pp. 142 y 153), enfatizaba Saussure.

En conclusión, para Saussure, tanto el significante como el significado son de naturaleza psíquica o mental, con lo que liberó de materialidad el signo lingüístico; y redujo su teoría del signo a una diada formalista. Saussure obvió también el carácter epistémico del signo. En ese aspecto, lo aventajó Peirce. A Louis Hjemslev correspondería enmendar al maestro.

\section{Una mirada a la teoría del signo lingüístico de Hjemslev}

El signo lingüístico es definido por Hjemslev (1984), en el capítulo xiII de su libro Prolegómenos a una teoría del lenguaje, como una entidad generada por la conexión entre una expresión y un contenido. La expresión y contenido son los funtivos que realizan la función de signo. Siguiendo la postura de Saussure, aclara que expresión y contenido son interdependientes (se presuponen necesariamente), por tanto, cada uno es una constante (siguiendo la terminología hjemsleviana). Hjemslev (1984, p. 73) discute lo que él llama el punto de vista tradicional, que considera el signo como una expresión que señala algo que está fuera de sí mismo (punto de vista monista); y el punto de vista de Saussure, que considera que el signo es una entidad generada por la conexión entre una expresión y un contenido (punto de vista diádico). Luego propone que tanto el plano del contenido (significado para Saussure) como el plano de la expresión (significante para Saussure) están compuestos a su vez por una sustancia y una forma. Aunque él no esquematizó su teoría del signo, esta ha sido representada de variadas maneras; una de las más comunes es esta: 


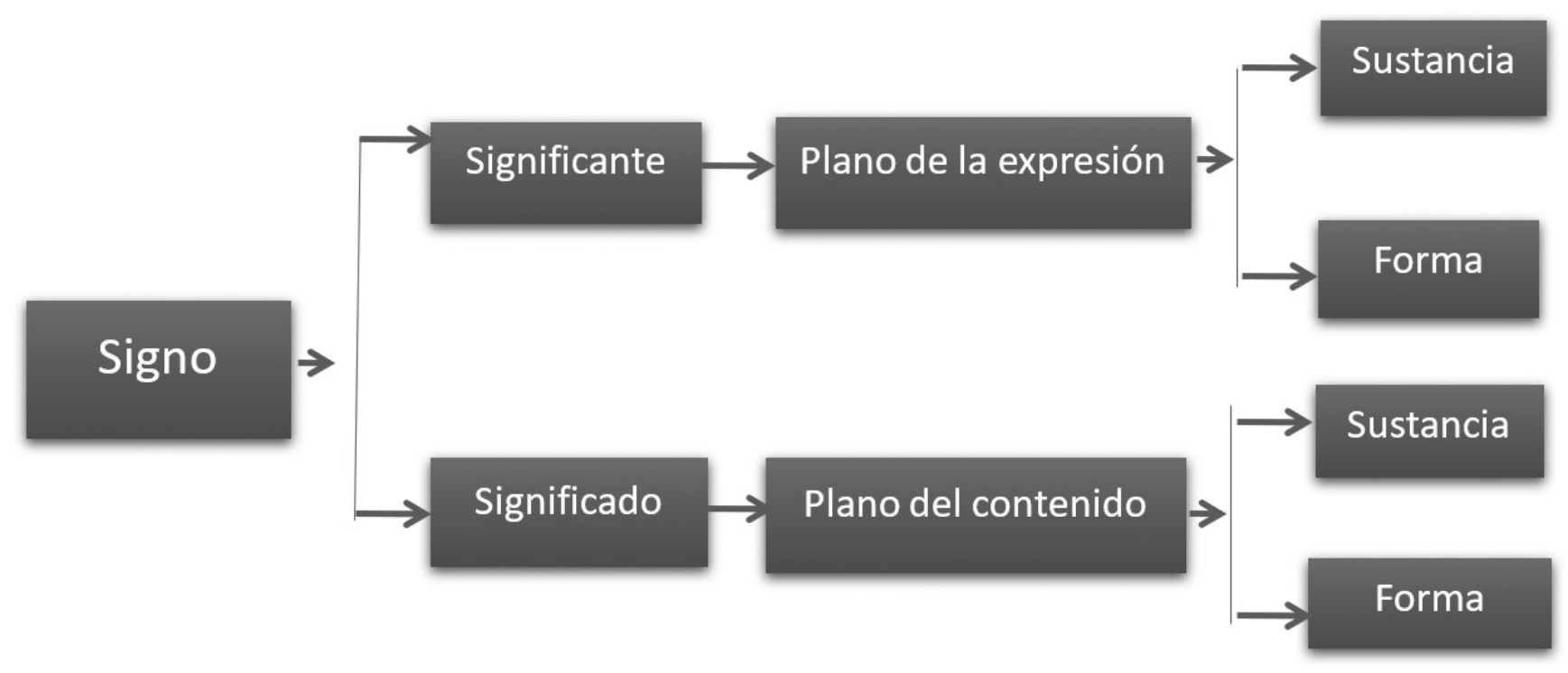

Figura 3. Diagrama de la teoría del signo de L. Hjemslev

Fuente: reelaboración propia.

Es palmaria la intención de Hjemslev de desdoblar los planos del signo propuestos por Saussure, dos unidades indisolublemente ligadas.

Si pensamos sin hablar, el pensamiento no será un contenido lingüístico ni funtivo de una función de signo. Si hablamos sin pensar, valiéndonos de una serie de sonidos a los que nadie que los escuche pueda concederles contenido alguno, tal habla será abracadabra, y no una expresión lingüística ni funtivo de una función de signo. (Hjemslev. 1984, p. 75)

A primera vista parecería que cada plano tiene a su vez un componente material (la sustancia) y otro formal o abstracto (la forma). Pero no es así. A menudo, los términos en Hjemslev son engañosos. En su teoría del signo, tanto la sustancia como la forma son formales. La forma de los dos planos tiene un carácter abstracto. Son categorías relacionales que constituyen tanto el plano de la expresión como el plano del contenido de las unidades de una determinada lengua. En cambio, la sustancia es las manifestaciones específicas de las unidades formales. Para Eco (1998, p. 72), Hjemslev sugiere un continuum de la expresión y un continuum del contenido.

La sustancia del plano de la expresión se relaciona estrechamente con el aspecto material o fonético de los sonidos (sentido según Hjemslev).

...Podemos hablar de un sentido de la expresión, sin que lo poco corriente de una afirmación semejante puede impedírnoslo. Los ejemplos que hemos dado (el continuum de vocales y de corte medio de la boca) serán entonces las zonas fonéticas del sentido formadas de modo diferente en las distintas lenguas, según las funciones específicas de cada lengua, u ordenadas de acuerdo con la forma de la expresión como sustancia de la expresión (Hjemslev, 1984, pp. 83-84).

En otras palabras, la fonética articulatoria sería la responsable de producir la sustancia, materia o sentido del plano de la expresión: fonemas, alófonos y sonidos. Para Hjemslev, las palabras homónimas 
tendrían mismo sentido de la expresión (sustancia fónica) pero diferente sentido del contenido (campo semántico).

La forma de la expresión es una constante, es específica en cada lengua, puesto que constituye las reglas de relacionarse la sustancia de la expresión. Así, las reglas de relacionarse o combinarse los fonemas para producir sílabas, las sílabas, para producir palabras; y las palabras, para producir sintagmas, etc. son constituyentes de la forma de la expresión. Hjemslev parece olvidarse del concepto de huella o imagen acústica propuesto por Saussure para definir el significante. Sin embargo, se aproxima un tanto a esta idea al afirmar que:

Cuando una persona familiarizada con el sistema funcional de una lengua dada (por ejemplo, la lengua materna) ha percibido un sentido del contenido o de la expresión, lo formará en esa lengua. Una parte esencial de lo que la gente entiende por "hablar con acento" consiste en dar forma, de acuerdo con las predisposiciones sugeridas por los hechos funcionales de la lengua materna del hablante, a un sentido de la expresión percibido (Hjemslev, 1984, p. 85).

Un sentido de la expresión funcionaría aquí como un organizador sígnico de las percepciones fonéticas o sustancia de la expresión.

En la concepción hjemsleviana del plano de la expresión del signo lingüístico, se enfatiza el carácter sensorial del mismo, en detrimento del carácter psíquico que planteó Saussure.

La sustancia del contenido, también llamada por Hjemslev sentido del contenido es la materia o «masa amorfa» de Saussure, ordenada por las divisiones determinadas por las formas. Hjemslev pone los ejemplos de los adverbios de lugar, aquí, ahí, allí, acá, allá. La noción de lugar sería la materia; la formación del paradigma sería la forma. Lo mismo aplica para el espectro de los colores, para el campo semántico de carne, etc. Diría Hjemslev que el sentido del contenido o materia sería el mismo, pero que la forma es distinta, porque el sentido de la sustancia es una variable (en términos suyos), mientras que la forma es una constante (también en términos suyos, equivalente a funtivo imprescindibles para que se produzca una función o relación).

El sentido o sustancia del contenido se aproxima bastante al referente, realidad o simplemente objeto del signo postulado por Peirce. "La materia, el continuum de que hablan los signos y mediante el cual hablan los signos, es siempre la misma: es el Objeto Dinámico de Peirce..." (Eco, 1998, p. 72). Hay que decir que es solo una aproximación, pues en ocasiones, Hjemslev la hace equivalente a pensamiento, con lo que vuelve a tomar distancia del objeto del signo. "En una ciencia que evita postulados innecesarios no hay base para afirmar gratuitamente que la sustancia del contenido (pensamiento) y la sustancia de la expresión (cadena de sonidos) preceda a la lengua en el tiempo..." (Hjemslev, 1984, p. 76).

La forma del contenido es una constante que consiste en la red de relaciones que clasifican, segmentan u organizan la materia o sentido del contenido de un modo particular en cada lengua. Por ejemplo, los paradigmas en que se organizan los campos semánticos pueden variar de una lengua a otra, como señalé anteriormente. Lo mismo se puede decir de la morfología y de la sintaxis de cada lengua.

Puede decirse que un paradigma de una lengua y otro correspondiente en otra lengua cubren una misma zona de sentido, la cual, aislada de esas lenguas, es un continuum amorfo sin analizar, en el que se establecen los límites por la acción conformadora de las lenguas (Hjemslev, 1984, p. 79).

La forma del contenido para Hjemslev, sirve de modelo para la organización de la materia, «masa amorfa», realidad u objeto del signo. "Igual que la 
misma arena puede colocarse en moldes diferentes y la misma nube adoptar cada vez una forma nueva, así también el mismo sentido se conforma o estructura de modo diferente, en diferentes lenguas."
(Hjemslev, 1984, p. 79). Una representación esquemática de los componentes de cada cara de los dos planos del signo de Hjemslev sería como ilustro en la figura 4.



Figura 4. La forma y la sustancia de los planos del signo lingüístico

Fuente: elaboración propia.

Nótese que en la teoría del signo de Hjelmslev, el objeto, referente o realidad está formalizado, por lo que la línea que separa la sustancia y la forma del plano del contenido no siempre está clara. Aunque su teoría del signo parece reivindicar el aspecto material del significante y del significado propuestos por Saussure, en realidad no es así, puesto que el objeto o referente del signo se pierde en las redes formales. Se diferencia de la teoría de Peirce en ese último aspecto y en el desdoblamiento del representamen o significante. Se demarca de la teoría de Saussure por la reivindicación de la cara sensorial del significante, por su intento de reivindicar un objeto material para el signo y porque minimizó tanto la imagen mental como la acústica.
Sin duda, Hjemslev abrió una perspectiva importante para estudiar el signo más allá de la diada saussureana de significado y significante. Su propuesta de cuatro funtivos para el signo lingüístico: forma del contenido y forma de la expresión y sustancia del contenido y sustancia de la expresión pudo haberlo conducido a la formulación de una teoría materialista-mental para el signo lingüístico. Se vio limitado para asumir esa visión por su lealtad al principio de inmanencia ("la lengua debe estudiarse en sí misma y por sí misma”) y a la máxima formalista ("la lengua es forma, no sustancia") ambas de Saussure. Por eso elude asumir explícitamente el objeto del signo propuesto por Peirce, mientras le asigna a la forma la categoría de una constante. No obstante, 
su propuesta representa un aporte extraordinario a la semiótica.

\section{Una mirada a la teoría del signo lingüístico de Charles Morris}

Charles Morris, enfocado más en las relaciones del signo que en la naturaleza del signo mismo, formula una teoría tetrádica, por lo que vale la pena revisarla. Sostiene este autor que:

En el proceso de semiosis implica tres (o cuatro) factores: lo que actúa como signo, aquello a que el signo alude, y el efecto que produce en determinado intérprete en virtud del cual la cosa es cuestión es un signo para él. Estos tres componentes de la semiosis pueden denominarse, respectivamente, el vehículo sígnico, el designatum, y el interpretante; el intérprete podría considerarse un cuarto factor. (Morris, 1994, p. 27)

Es evidente la relación de esta teoría con la Peirce, aunque Morris no lo cite. Nótese que el vehículo sígnico es exactamente el representamen, el designatum es el objeto del signo y el interpretante es exactamente el mismo de Peirce. En esta propuesta, hay un elemento importante ausente en las tres teorías anteriores. Se trata del sujeto del proceso de cognición o semiosis, al cual Morris llama intérprete, y luego define (una página más adelante) cómo el agente del proceso.

La incorporación del sujeto del conocimiento como parte del proceso de semiosis es una enmienda importante a las semióticas de Peirce, de Saussure y Hjemslev, pero no se puede asumir que agrega un nuevo elemento al signo, sino a la semiosis. Parece obvio que el conocimiento lo construyen los sujetos del conocimiento, para lo cual elaboran signos, pero cancelarlo puede obedecer a una epistemología de impersonalización del proceso cognitivo. Por eso, Morris insiste:
Algo es un signo, si y solo si, algún intérprete lo considera signo de algo; la consideración de algo es un interpretante solo en la medida en que es evocado por algo que funciona como signo: un objeto es intérprete solo si, mediatamente, toma en consideración algo. (Morris, 1994, p. 28)

Su énfasis en correlacionar los elementos de la semiosis recuerda el empeño de Saussure en establecer una relación dialécticamente indisoluble entre significante y significado.

Ahora bien, el hecho de que Morris proponga cuatro elementos en el proceso de semiosis no implica que su teoría del signo sea tetrádica. Lo que es tetrádica es la semiosis, pero el signo es un solo elemento de esa tétrada, por lo que este en sí mismo es una mónada, como se puede verificar en las dos citas anteriores.

\section{Mi propuesta}

Aunque se dice que la teoría del signo de Peirce es triádica, en realidad no es así. Para este autor, signo es el representamen, el cual en el proceso de semiosis entra en relación con el objeto y con interpretante. Su aporte a la teoría del signo fue incluir el interpretante (significado para Saussure) como mediador entre el signo y la percepción de la realidad. En Peirce, el signo es una mónada al igual que en Morris; y el proceso de semiosis, una triada.

En la teoría del signo de Saussure está implícita la idea de que tanto el significante como el significado tienen una cara material y otra mental. El acierto de Saussure fue insistir en el aspecto psíquico del significante, pero soslayóla cara material o físico del significante, es decir, los sonidos específicos equivalente al sentido de la sustancia del plano de la expresión de Hjemslev. De manera que, la teoría del signo de Saussure es diádica y psíquica, pues no toma en cuenta el aspecto material del 
signo lingüístico: ni los sonidos físicos, específicos o fonéticos del significante; ni el objeto, realidad o «sustancia amorfa» del significado.

Hjemslev hizo un esfuerzo importante para incorporar el aspecto material de los dos planos psíquicos de la teoría del signo saussureano, con lo que se acercó a superar la visión mentalista del maestro. La sustancia del plano de la expresión reivindica el aspecto fonético del signo, obviado en Saussure. Sin embargo, el aspecto mental del significante que correspondería a la forma del plano de la expresión no está muy explícito. En cuanto al plano del contenido, da la impresión, antes de profundizar en la comprensión de la teoría, de que se trata de la reivindicación del objeto o referente del signo, pero en realidad es de la formalización del concepto significado de Saussure. La sustancia del plano del contenido (llamada también, sentido de la sustancia del contenido) es un tanto confusa, pues en ocasiones se refiere a ella con si fuera el pensamiento, con lo que coincidiría con la forma del contenido; y en la mayoría de las ocasiones, como equivalente de un campo semántico, con lo que se aproxima al objeto peirciano y la "masa amorfa», de Saussure. En otras palabras, la teoría del signo lingüístico de Hjemslev atenúa el aspecto material o sensorial del significante (imagen acústica) y el objeto o referente del signo.

Por su parte, Morris propone que el signo establece una relación tetrádica en el proceso de semiosis. El elemento demarcativo de Morris con respecto a las demás teorías es la intervención de un agente de la semiosis al cual llama interpretante.

Mi propuesta es que el signo tiene dos planos (significante y significado), como propuso Saussure; que tanto el significante como el significado son diáticos, como lo concibió Hjemslev; y que tanto el significante como el significado tienen una cara mental o psíquica y sendas bases materiales, a partir de las cuales se construyen. Mi diferencia con
Hjemslev es que este se quedó en el formalismo y no planteó con claridad el aspecto fonológico, abstracto o mental del significante, como lo hizo Saussure; y que obvió el objeto del signo, como lo planteó Peirce. En mi propuesta, los sonidos específicos, activan las «huellas mentales» compuesta de fonemas. Por ejemplo, los fonemas tienen realizaciones indefinidas, pero en la mente de los hablantes de una lengua hay solo una representación de esas variaciones. Cada variación de un fonema funciona como un activador de esa representación. Lo propio sucede con las palabras. Estas tienen una variedad indefinida de pronunciaciones o realizaciones, pero cada hablante tiene una representación fonológica de cada una, la cual es activada con la pronunciación de cualquiera de sus variaciones. En otras palabras, el significante tiene el aspecto fonológico que señaló Saussure y un aspecto o cara fonética que son las realizaciones de la cara fonológica.

Lo mismo postulo para el plano del contenido o del significado. Los significados se construyen a partir de la actividad cognoscitiva del sujeto cognoscente. Este sujeto cognoscente para producir significados o conceptos necesita realizar abstracciones de la realidad, objeto o «masa amorfa». Por tanto, una epistemología del signo lingüístico no puede obviar una relación entre sujeto cognoscente, objeto y concepto. La actividad del sujeto cognoscente se puede subsumir en los significados, puesto que estos presuponen ese sujeto. En esa tesitura, el plano del contenido de Hjemslev o del significado, de Saussure, tendría dos caras: una mental (los conceptos) y otro material (la realidad u objeto del signo).

En síntesis, el signo lingüístico tiene los planos de significante y significado, propuestos por Saussure; pero estos a su vez tienen dos caras: sonidos o activadores sígnicos e imagen acústica, el significante; y concepto y objeto, el significado, por lo que se puede reivindicar el carácter biplánico del signo y la cara mentalista de esos planos propuestos por Saussure; pero también reivindico una cara material para para ambos planos (ver figura 5). 




Figura 5. Planos y caras del signo lingüístico

Fuente: elaboración propia.

La psicolingüística ha comprobado el acierto de Saussure al postular una imagen acústica como parte del signo lingüístico. Este acierto de Saussure se ha soslayado a pesar de las implicaciones que tiene para la enseñanza de la lengua y para la comunicación lingüística. La imagen acústica se constituye en un arquetipo o representación que permite percibir y aprehender el vehículo sígnico, constituir el significante del signo y activar una representación conceptual para completar el proceso de semiosis. Presentaré, a continuación, algunas evidencias de la importancia de ese componente del signo.

Las palabras tienen un umbral de activación o encendido de la imagen acústica del significante, el cual depende del contexto sintáctico y mnemotécnico, de la frecuencia o recurrencia de la palabra, del punto de unicidad y de la concurrencia de las palabras, entre otros factores.

Este umbral de activación o encendido es la información o segmento lexical que se necesita oír o leer para activar la imagen mental o arquetipo fonoló- gico del signo lingüístico. Así, cuando conversamos o leemos, con frecuencia «adivinamos» el final de la palabra sin oírla o leerla completa, como hacen los algoritmos de los teléfonos móviles; y Facebook, cuando marcamos nombres de contactos ya registrados. Obsérvese que, entre todas las posibilidades, los algoritmos eligen las más recientes o las más frecuentes. En una conversación en el seno de una familia con miembros vinculados a la universidad, cuando uno de los participantes en la conversación escucha el segmento UNI, activa la palabra UNIVERSIDAD, como si la hubiera escuchado completa. Incluso, el hablante o el lector puede inferir o reconocer los componentes léxicos (fonemas y grafemas) con mucho más facilidad y rapidez en el contexto lexical (efecto palabra) que “...en una serie aleatoria de letras" (Cueto, 2002, pp. 26-27).

Las palabras (signo lingüístico) también tienen punto de unicidad. Este descubrimiento llevó al psicólogo británico John Morton postular su modelo de acceso directo al léxico, llamado logogén (citado por Cuetos, 2020, pp. 33-38). El punto de 
unicidad es el segmento de la palabra a partir del cual no existen otras, por lo que se constituye en el mayor umbral de encendido o activación de la imagen acústica de un significante. Así, el adjetivo imperecedero tiene como punto de unicidad el segmento impere; el adverbio monstruosamente, el segmento monstruoso; y el sustantivo obscenidad, el segmento obsceni.

Mientras el umbral de encendido depende de la experiencia de cada hablante; el punto de unicidad es totalmente objetivo, porque el hablante no tiene opción de activación. Claro, si el hablante no conoce la palabra a la que se refiere el segmento léxico (oral o escrito) tendrá que esperar a leer o escuchar la palabra completa. Incluso, puede ocurrir que la distorsione, al confundirla con otra parecida fonética o gráficamente. Por eso, mientras mayor es el léxico de un hablante, más rápida será la percepción de las palabras, y por tanto su lectura o audición. En un dictado que realizamos a un grupo de 18 doctorandos, encontramos que mientras menos frecuente era una palabra, mayor era la dificultad para escucharla y retenerla. Por ejemplo, la palabra nenúfares, 07 (38.89\%) la retuvieron; 08 (44.44\%) no retuvieron nada, o sea, se les «evaporaron» los sonidos; 01 (5.5\%) creyó escuchar «menúfares»; 01 (5.5\%) creyó escuchar «entre pared y piedras»; 01 (5.5\%) creyó escuchar «menú animal». Cuando les pregunté a los participantes si conocían la palabra nenúfares, ninguno de los que la «escucharon» fonéticamente la habían oído antes; y cinco de los que activaron la imagen acústica correspondiente ya la conocían.

Con la palabra otorrinolaringología se produjo un resultado opuesto. Todos la escucharon fonológicamente y la escribieron bien, a pesar de ser más extensa y más difícil de pronunciar que nenúfares. El nivel doctoral de los participantes hace suponer que ya conocían esa palabra, es decir, que la tenían registrada fonológica y ortográficamente, por lo que, incluso, si yo la hubiera pronunciado mal, la habrían escuchado como ya la tenían registrada en su memoria fonológica.

\section{Conclusión}

El signo es un concepto fundamental, no solo para la lingüística, sino para todas las ciencias humanísticas. Todos los procesos cognitivos están basados en el signo, especialmente en el lingüístico. De ahí que una teoría del signo tiene repercusiones en varias disciplinas, entre ellas, la semiótica, la logopedia, la epistemología, la psicología de la percepción y la filosofía.

A pesar del esfuerzo de Saussure y de Hjemslev por reivindicar el carácter biplánico del signo, los semiólogos, los filósofos y los lingüistas lo reducen al aspecto material del significante, unas veces de manera explícita; y otras, implícitas. Mi propuesta toma de Peirce el objeto o referente del signo; de Saussure, su énfasis en el carácter mental tanto del significado como del significante; y de Hjemslev, su intento por reivindicar el carácter material tanto del significante como del significado, pero la complementa con la reivindicación de la noción que hace Hjemslev para ambos planos. Es decir, el signo no es un habitante de Planilandia, como los objetos unidimensionales de la novela de ese mismo nombre (Abbott 1999); sino en Esferilandia, como los objetos de varios planos.

El concepto de activador sígnico que propongo para los sonidos, aspecto sensorial o la sustancia del significante, implica que este no tenga que ser exclusivamente fónico, pues la imagen acústica o logogén podría ser activada por "manifestaciones» o estímulos sensoriales de otra índole, como sucede en la lengua escrita. También implica que las percepciones están condicionadas no solo por el interpretante propuesto por Peirce, sino también por la imagen acústica planteada por Saussure.

Mi propuesta impacta la percepción, pues el signo es concebido como el producto de una cadena de activaciones: el objeto material del significante (los sonidos), activa la imagen acústica; la imagen acústica, activa la imagen mental o interpretante de Peirce; la imagen mental activa o recrea la experiencia empírica del intérprete, la cual, a su vez, remite a la realidad objetiva. 
La relación del signo lingüístico, como la de todos los signos simbólicos, es triádica, como lo postuló Peirce. Específicamente, se relaciona con el intérprete de Morris, y con la realidad, «masa amorfa» u objeto, como propuso Peirce.

\section{Referencias}

Abott, E. (1999). Planilandia: una novela de muchas dimensiones. Barcelona, España: Torre de Viento.

Cuetos, F. (2002). Psicología de la lectura. Barcelona: Praxis.

Eco, U. (1976). Signo. Barcelona: Labor.

Eco, U. (1998). Semiótica y filosofía del lenguaje. Barcelona: Lumen.

Hjemslev, L. (1984). Prolegómenos de una teoría del discurso. Madrid: Gredos.
Malmberg, B. (1974). Lingüistica estructural y comunicación humana. Madrid: Gredos.

Morris, Ch. (1994). Fundamentos de la teoría del signo. Barcelona: Paidós.

Peirce, Ch. S. (1953). Collected papers (9 vols). Massachusetts: Harvard University Press.

Peirce, Ch. S. (1973). Semiótica de Peirce. Buenos Aires: Ediciones Nueva Visión

Peirce, Ch. S. (2007). La lógica considerada como semiótica: índice del pensamiento peirciano. Madrid: Biblioteca Nueva.

Peirce, Ch. S. (2014). La ciencia de la semiótica. Buenas Aires: Ediciones Nueva Visión.

Saussure, F. (1980). Curso de lingüística general. Madrid: Alianza Editorial.

\section{Datos de Filiación}

Bartolo García Molina. Doctor en Filosofía del Lenguaje por la Universidad Complutense de Madrid. Fue profesor en la Universidad Autónoma de Santo Domingo (UASD), desde 1982 hasta el 2008, en las asignaturas Historia de la lengua, Fonética y Fonología, Teorías Lingüística y de Morfosintaxis de la Lengua Española. En la actualidad es profesor de Teorías del discurso y de El discurso de la ciencia en varias maestrías en distintas universidades dominicanas. Su labor docente se vincula con el estudio e investigación permanente de áreas de Lingüística Aplicada, redacción, lectura, teoría del discurso y el discurso científico. 